\section{Case reports of observed significant improvement in patients with ARDS due to COVID-19 and maximum ventilatory support after inhalation of sodium bicarbonate}

\author{
Wardeh A*, Conklin J and Ko M \\ Department of Critical Care Medicine, Uniontown Hospital, Uniontown, PA, USA
}

\section{Introduction}

Theemergence ofCOVID-19worldwideinanunprecedented pandemic. COVID-19 has a significant mortality, mostly from acute lung injury. We reviewed the available literature from China and Europe in regard to the behavior of SARS-Cov2 and ability to adhere to the cell wall $[1,2]$. The evidence based literature describes three component for the virus to grant entry to the target cells including Cathepsin B/L (the viral cap protein needed for initial connectivity to the cell wall), the angiotensin converting enzyme 2 and a low PH environment to allow the first connectivity of the virus to the cell wall [3]. The goal of our Case study was to prevent SARS- SARS-Cov2 from entering target cells by raising the airways $\mathrm{PH}$ using sodium bicarbonate inhalation. The sodium Bicarbonate inhalation (4.2\% concentration) has been used safely in Cystic fibrosis (CF) patients with inspissated mucoid impaction [3,4] and in chloride inhalation toxicity by opposing the effect of the low PH induced by the insulting agent [4,5]. It has not been administered for COVID -19 patients particularly prior to this study.

\section{Methods}

Four patients required mechanical ventilation due to COVID-19 pneumonia. All patients have proven positive COVID-19 RNA collected from the airway's secretions. Each one of the patients received inhaled $4.2 \%$ sodium bicarbonate as a salvage therapy. All four patients had severe adult respiratory distress syndrome (ARDS) and required $100 \%$ Fraction of inspired oxygen (FIO2). Due to severe hypoxia despite 100\% FIO2, these patients were placed on Positive end expiratory pressure (PEEP) of 15 to maintain their Oxygen saturation at $87 \%$ at minimum. Also, a standard PEEP of 15 was used throughout the process to keep the alveoli patent to

\section{More Information}

*Address for Correspondence: Anas Wardeh, Department of Critical Care Medicine, Uniontown Hospital, Uniontown, PA, USA,

Tel: 508-479-4213; Email: awardeh1@aol.com; awardeh1@icloud.com

Submitted: 11 May 2020

Approved: 18 May 2020

Published: 19 May 2020

How to cite this article: Wardeh A, Conklin J, Ko M. Case reports of observed significant improvement in patients with ARDS due to COVID-19 and maximum ventilatory support after inhalation of sodium bicarbonate. J Clin Intensive Care Med. 2020; 5: 016-019.

DOI: 10.29328/journal.jcicm.1001029

Copyright: @ 2020 Wardeh A, et al. This is an open access article distributed under the Creative Commons Attribution License, which permits unrestricted use, distribution, and reproduction in any medium, provided the original work is properly cited.

Keywords: Medical emergency service; Ambulances; Optimization; Simulation; Response time

\section{A) Check for updates}

OPEN ACCESS

assure the delivery of the nebulized sodium bicarbonate via the ventilator circuit.

Two of the patients underwent a bronchoscopy to exclude airway obstruction (Case 1 and Case 4) and one patient initially received instillation of sodium bicarb of $40 \mathrm{ml}$ in an increment of $2 \mathrm{ml}$ in each segment during the bronchoscopy to dislodge mucous plugs. (These two patients who underwent bronchoscopy, were the sickest patients with APACHE score higher than 25).

Our patients were treated with nebulization of sodium bicarbonate $4.2 \%$ solution. The dose of sodium bicarbonate used was $10 \mathrm{ml}$ of the nebulized solution and was delivered via the inspiratory limb of the ventilator tubing every 6 hours. The FIO2 then reduced by 10\% every 4-8 hours to keep oxygen saturation at $>88 \%$ as tolerated. Once the FIO2 is down to $50 \%$, the PEEP was reduced by 2.5 every 6-12 hours to keep the oxygen saturation $>88 \%$. We obtained a daily chest $x$ rays on every patient to evaluate the effect of the treatment radiologically. Arterial blood gas (ABG) was also obtained daily to evaluate for the development of Hypercarbia and monitor the arterial blood PH. We applied ARDSnet Lung protective strategy (a well-known guidelines for ventilator management in ARDS) on all of the cases. The treatment was discussed with 
the family members over the phone due to the hospital's no visitation policy and the families were in agreement with the application of sodium bicarbonate inhalation therapy. Also, with the support of the pharmacy staff at our institution, the dose was addressed to be comparable to the doses used for mucoid impaction in cystic fibrosis (CF) patients. The cases were reviewed daily and assessed clinically and radiologically. The arterial blood gases were reviewed daily to monitor for possible metabolic alkalosis, which did not occur in any of the patients.

\section{Cases}

\section{Case 1}

49 year old female with PMHx significant for partial thyroidectomy many years ago, none smoker life time, no history of asthma, previous PFTs were normal, BMI > 35, worked as a teacher, no history of travel exposure. She presented to the hospital with fever, nonproductive cough, severe dyspnea and altered mental status. On arrival, her 02 saturation was $60 \%$ on room air. Her oxygen saturation responded to high flow 02 to $95 \%$ for the first two days. Initially, she improved but then has to be intubated due to desaturation. She was placed on a 100\% FIO2 and PEEP of 12 to keep her 02 saturation at $88 \%$. She tested positive for COVID -19. Her CXR showed diffuse infiltrates. Four days after intubation, she was started on sodium bicarbonate $4.2 \%$ inhalation every 6 hours. Thirty six hours later, she was able to be weaned off the ventilator and extubated to NC oxygen at 4 LPM and now she is on RA with 02 saturation 95\%. Post extubation she continued to have mild delirium (Figure 1a,b).

\section{Case 2}

60 year old male with a history of hypertension on amlodipine and Enalapril, $\mathrm{He}$ and his wife have been tested positive for COVID -19 via a clinic as an outpatient. His wife self-quarantined at home, while he developed dyspnea and intractable cough. He presented to the hospital with Oxygen saturation of $49 \%$ on RA. Started on a high flow 02 . He declined intubation initially for the first 2 days of hospitalization. He was competent to make his own decision. After 2 days, he agreed to intubation. He was placed on AC $6.5 \mathrm{ml} / \mathrm{kg} / \mathrm{VT}$ and PEEP of 15 with FIO2 of $100 \%$. Oxygen saturation post intubation
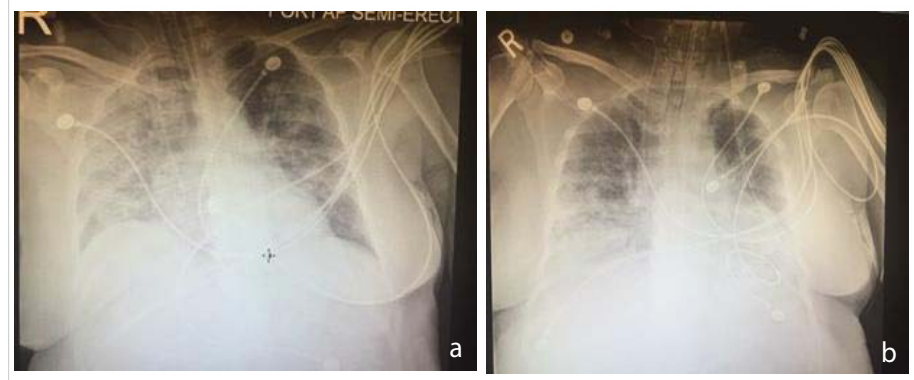

Figure 1: a) Chest $x$ ray (Case 1) showing extensive infiltrate, largely on the right, b) imaging for the same patient, After sodium bicarb treatment. Noted. was $89 \%$. He was started on sodium bicarbonate inhalation every 6 hours. After 48 hours, his ventilatory requirement improved, his 02 saturation increased to $98 \%$ on $40 \%$ FIO2 and the PEEP reduced to 5 . He was extubated to NC at 4 LPM. And discharged to the regular ward (Figure 2a,b).

\section{Case 3}

65 year old male traveling from California to attend a funeral for his father in law. He has a history of hypertension only. He presented with increased dyspnea and nonproductive cough, along with his wife (Case 4). His 02 saturation was $72 \%$ on RA, he responded to a high flow 02 and $100 \%$ nonrebreather. He refused intubation for the first 7 days due to the believe that intubated patients do not survive. Finally, he agreed to the intubation. He required $100 \%$ FIO2, PEEP of 15 and required prone positioning for two consecutive days. He was started on the above Sodium Bicarbonate protocol and on day number 3 of sodium bicarbonate treatment was able to reduce his FIO2 to $50 \%$ and PEEP reduced to 10 . He tolerated spontaneous breathing trial for 30 minutes but failed due to agitation. He developed thrombocytopenia and health care acquired pneumonia as well. The DIC work up was negative. He is expected to be extubated in the next 2-3 days (Figure 3a,b).

\section{Case 4}

63 year old female, the wife of Case 3, who was in the same funeral. She is a retired ICU nurse, traveling from California, presented with acute respiratory failure. Initially, she elected to use noninvasive measures with High flow oxygen and $100 \%$

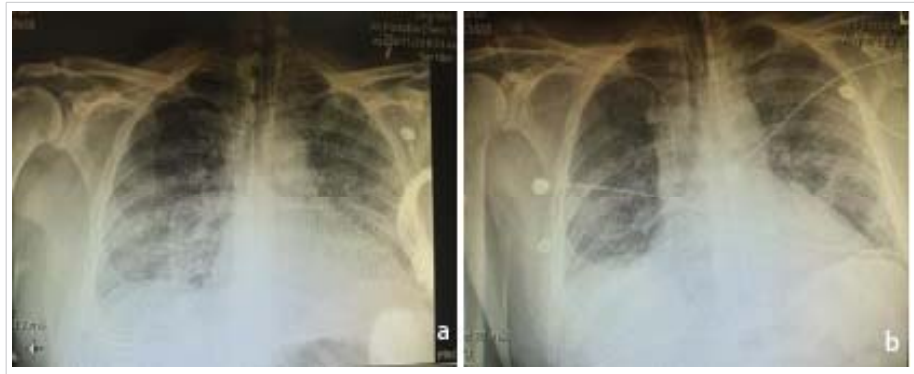

Figure 2: a) Chest $x$ ray (Case 2) prior to sodium bicarbonate inhalation, Showing bibasilar diffuse infiltrates, b) Chest $x$ ray (Case 2 ) 3 days after, Sodium bicarbonate inhalation, showing improved Infiltrates at the lower lobes.
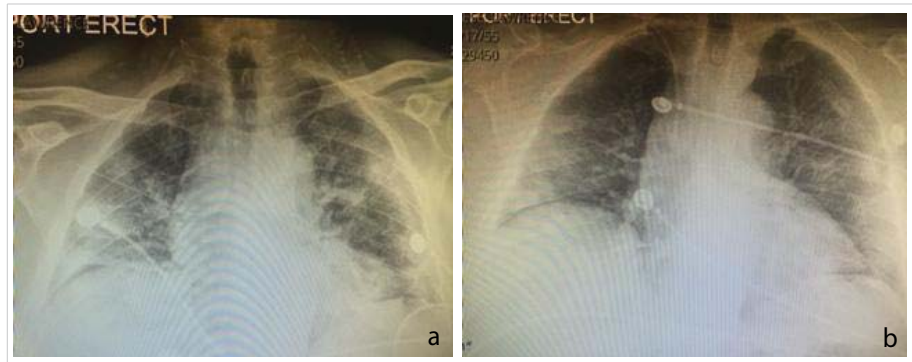

Figure 3: a) Chest $x$ ray prior to sodium bicarb inhalation. (Case 3), multiple bilateral infiltrates shown just before intubation, the patient declined intubation for few days, then agreed to it. b) two days after inhalation of sodium bicarbonate. The patient is extubated with significant improvement of the infiltrates mostly on the right. (Case 3 ). (Chest $x$ ray post successful extubation). 
nonrebreather. After six days, she felt tired and agreed to the intubation. She was placed on $100 \%$ FIO2 and 15 of PEEP. She underwent a bronchoscopy and instillation of a total of $40 \mathrm{ml}$ of sodium bicarbonate in increments of $2 \mathrm{ml}$ per segment. She did not have significant amount of secretions. On day 2 post treatment with inhaled sodium bicarbonate, she improved and her 02 requirement was down to $50 \%$ and PEEP of 8 . She is expected to be extubated in the next 24-48 hours. She did not show multiorgan system disease (Figure 4a,b).

\section{Results}

Our case series included four patients treated with sodium Bicarbonate inhalation protocol while been on the ventilator in coordination with the ARDSnet ventilator management as stated above [6]. All improved clinically and radiologically and two Patients proceeded to extubation within 24-72 hours. The other two patients showed improvement but could not be extubated at the time of writing this article. However, they are expected to be extubated in 24-48 hours. Of note, these two patients declined intubation for several days prior to agreeing to it. Nonetheless, both are undergoing Spontaneous breathing trial at this time. These two patients who remains intubated after 72 hours, required pronation initially for the first 48 hours only. However, their Chest imaging showed significant improvement, (Firgure $3 \mathrm{~b}$ and Figure $4 \mathrm{~b}$ ). The $\mathrm{PH}$ measured by arterial blood gas did not change with the sodium bicarbonate inhalation treatment. The $\mathrm{pH}$ on the ABG was ranging between 7.29 to 7.38. One patient developed hypercarbia possibly due to high PEEP.

Two of our patients (Case 1 and Case 2) were successfully extubated and transferred out of the ICU after 48 hours of starting the sodium bicarbonate inhalation. One of them remains on nasal cannula oxygen at 2-4 LPMand is being treated on the regular COVID -19 ward. None of the patient treated with sodium bicarbonate inhalation required escalation in ventilator support such as re-intubation, prone positioning or increased oxygen requirement. One patient developed Health Care acquired pneumonia which delayed his liberation from the ventilator. He also developed thrombocytopenia thought to be due to sepsis, unrelated to COVID-19.

\section{Discussion}

Although alkalization of the airways by raising the $\mathrm{PH}$
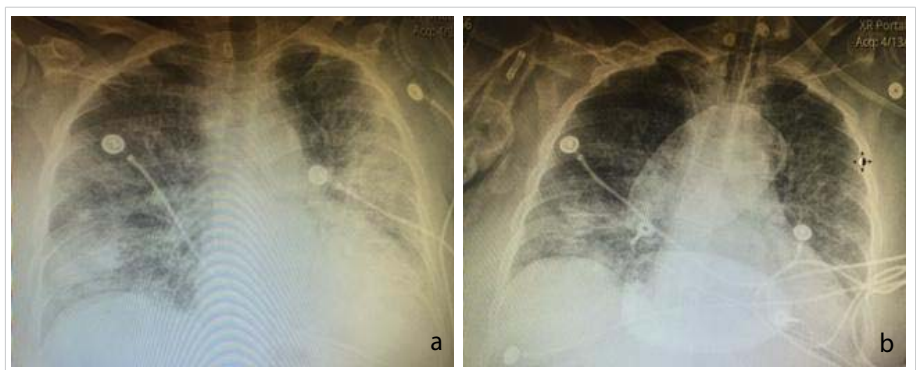

Figure 4: a) (Case 4) chest $x$ ray showing extensive. Infiltrates diffusely due to COVID-19. b) (Case 4), near complete resolution Of the left lung infiltrate. using sodium bicarbionate inhalation has been studied in cystic fibrosis patients with mucoid copious secretions as the higher $\mathrm{PH}$ aims at reducing the viscosity of the secretions in this patient population $[3,4]$. The sodium bicarbonate inhalation also used as a salvage therapy after toxic inhalation with Phosgene [5], it has not been tested for COVID -19 pneumonia. Our aim was to alter the alveolar PH in an effort to prevent further inoculation of the coronavirus into the lung parenchyma [4].

In the past, the literature coming out of China showed altering the $\mathrm{PH}$ could prevent the connectivity of the SARSCov2 to the cell wall $[1,7]$. The PH of the sodium Bicarbonate of $4.2 \%$ is known to be 8.4. Although this $\mathrm{PH}$ is much higher than the serum $\mathrm{PH}$, using it in inhalation form has proven to be safe without altering the $\mathrm{PH}$ of the blood [4,5]. Several studies are entertaining the treatment of the current COVID -19, mostly involving the development of a vaccine or a drug treatment, however, no study has looked at treating COVID -19 by altering the airways $\mathrm{PH}$ using sodium bicarbonate inhalation. Our case series showed an improvement in respiratory status and survival in all four patients who were studied. The time from treatment to liberation from the ventilator was surprisingly short at 1-3 days in the first two cases and expected to be about 5 days in the last two cases (Case 3 and Case 4). These two cases declined intubation for the first few days (patient preference), and possible early intubation would have resulted in shorter ventilator time. Usually, similar patients with COVID-19 pneumonia and high APACHE score require longer ventilation time (mostly 10 - 21 days). These cases presented with extremely severe ARDS with mortality rate exceeding 90\% given their high APACHE score.

Although this is a case series and the observation cannot be generalized, we do believe that it is worth investigating the efficacy of inhaled sodium bicarbonate treatment on severely ill COVID -19 patients in a larger randomized controlled trial. Altering the $\mathrm{PH}$ of the airways could be an effective treatment in ARDS patients with COVID-19 pneumonia.

In addition, measuring the viral load in the exhaled breath pre and post treatment could be helpful to evaluate the response to airway alkalization.

Many institutions do not allow nebulization of medications in COVID-19 patients due to the risk of aerosolizing the virus and infecting the health care workers. This issue should be looked at in a large scale study with sodium bicarbonate, since the virus does not seem to survive a higher PH. In addition, the sodium bicarbonate inhalation was administered via a closed circuit in vented patients.

Furthermore, applying a $\mathrm{PH}$ prob via the endotracheal tube to test the airway $\mathrm{PH}$ can be used to monitor the relation between the viral load in the exhaled breath $\mathrm{PH}$ while on sodium bicarbonate inhalation which can be done in a larger trial In our series, we did not apply a PH prob due to the 
small number of cases we studied. Based on the above, early intubation and sodium Bicarbonate inhalation in intubated COVID-19 positive patients with severe ARDS appears promising but need to be evaluated in randomized controlled trial (RCT). An RCT could be easily conducted given the availability of the sample size in the current pandemic. Such a study will recognize the benefits, the short term outcome and the value of altering airways $\mathrm{PH}$ and identify the confounding factors.

\section{References}

1. Chu, V. McElroy, L. Chu, V. Bauman, B. Whittaker, G. The Avian Coronavirus Infectious Bronchitis undergoes direct low $\mathrm{PH}$ dependent Fusion activation during entry into the host cells. J Virol. 2006; 80 : 3180-3188.

PubMed: https://www.ncbi.nlm.nih.gov/pubmed/16537586

2. Yong $Z Y$, Huang $Y$, Ganesh L, Leung K, Kong wp. et al. pH-Dependent Entry of Severe Acute Respiratory Syndrome Coronavirus Is Mediated by the Spike Glycoprotein and Enhanced by Dendritic Cell Transfer through DC-SIGN. J Virol. 2004; 78: 5642-5650.

PubMed: https://www.ncbi.nlm.nih.gov/pmc/articles/PMC415834/

3. McShane D, Davies JC, Davies MG, Bush A, Geddes DM, et al. Airway surface PH in subjects with cystic fibrosis. Eur Respir J. 2003; 21: 37-42. PubMed: https://www.ncbi.nlm.nih.gov/pubmed/12570106

4. Gomez CCS, Parazzi PLF, Clinckspoor KJ, Mauch RM, Pessine FBT, et al, safety, tolerability and effectes of sodium bicarbonate inhalation in cystic fibrosis. Clin Drug Investig. 2019; 40: 105-117.

PubMed: https://www.ncbi.nlm.nih.gov/pubmed/31721070

5. Aslan S, Kandiş H, Akgun M, Cakir Z, Inandi T, et al. The effect of nebulized sodium bicarbonate treatment on RADS patients due to chlorine gas inhalation. Inhal. Tox. 2006; 18: 895-900.

PubMed: https://www.ncbi.nlm.nih.gov/pubmed/16864407

6. NIH NHLBI ARDS clinical network, mechanical ventilation protocol summary, 2017.

7. Kis A. Toth L, Kunos L, Gyorgy L, Wanner A. The effect of airway alkalinization by nebulized sodium bicarbonate on airway blood flow. European Res. Journal. 2012; 40: P2143.

8. Chinese SARS Molecular Consortium. Science 10.1126/ science.1092002.

9. Bosch BJ, Martina BE, Van Der Zee R, Lepault J, Haijema BJ, et al. Severe ARDS due to SARS CoV infection inhibition using spike protein heptad repeated-derived peptides. Proc Natl Acad Sci USA. 2004; 101: 8455-8460.

PubMed: https://www.ncbi.nlm.nih.gov/pubmed/15150417 